\title{
LAS FUNCIONES COMPETITIVAS DEL HUMOR EN LOS COTILLEOS DE JÓVENES VEINTEAÑERAS
}

\author{
VIRGINIA ACUÑA FERREIRA \\ Universidad de Vigo
}

Recibido: $15 / 09 / 2014$

Aceptado: 24/11/2014

\section{Resumen}

Las investigaciones sobre género y humor en la conversación espontánea señalan que las prácticas humorísticas de las mujeres en este tipo de contextos se caracterizan por sus rasgos cooperativos y su funcionalidad principalmente orientada al refuerzo de la solidaridad y/o a la subversión de normas y estereotipos de género. El objetivo de este artículo es resaltar las características formales y funcionales del humor que se produce dentro de la práctica de cotilleo, analizando fragmentos de dos conversaciones espontáneas entre estudiantes universitarias gallegas de poco más de veinte años. En contraste con la caracterización general del humor conversacional de las mujeres en la bibliografía, en este análisis se ponen de relieve las funciones competitivas que subyacen en este tipo de discurso y su papel en el mantenimiento y refuerzo de formas de capital simbólico femenino como la apariencia física y las relaciones sexuales/ románticas con los hombres.

Palabras clave: conversación, humor, cotilleo, estudiantes universitarias, funciones competitivas, capital social femenino, apariencia física, relaciones con los hombres.

\begin{abstract}
Studies on gender and humour in spontaneous conversation have noted that women's humorous practices in these contexts are characterized by their cooperative features and by their functionality primarily oriented to the reinforcement of solidarity and/ or the subversion of gender norms and stereotypes. The aim of this article is to illuminate the formal and functional characteristics of humour in the practice of gossip by analyzing extracts of two spontaneous conversations among Galician female undergraduate students who are in their early twenties. In contrast to the general
\end{abstract}


characterization of women's conversational humour in the literature, this analysis highlights the competitive functions of this kind of discourse and as its role in the maintenance and reinforcement of forms of female symbolic capital such as physical appearance and sexual/romantic relationships with men.

Keywords: conversation, humour, gossip, female undergraduate students, competitive functions, female symbolic capital, physical appearance, relationships with men. 


\section{Introducción}

Los chistes, las burlas, las historias divertidas o las salidas graciosas constituyen formas de crear humor que han estado estereotípicamente asociadas con el habla de los hombres en las culturas occidentales. Por ello, desde una perspectiva de género se ha destacado con frecuencia la visión general del humor como un tipo de discurso que está fuertemente ligado a la masculinidad y, en cambio, casi totalmente desvinculado de la feminidad; algunos de los primeros trabajos sobre lengua y género, como la conocida descripción de la «lengua de las mujeres» de Robin Lakoff ${ }^{1}$, reflejan y reproducen poderosamente esta idea, señalando incluso una falta de sentido del humor en las mujeres: «women can't tell jokes - they are bound to ruin the punch line, they mix up the order of things, and so on. Moreover, they don't «get» jokes. In short, women have no sense of humor ${ }^{2}$. Para explicar por qué el contar chistes es algo que se asocia con los hombres, Lozano Domingo ${ }^{3}$ señala que esta tarea discursiva conlleva asumir un papel protagonista y que esto no es compatible con el comportamiento «discreto» que se espera de las mujeres en muchos acontecimientos comunicativos. Por otra parte, el carácter competitivo que presentan determinadas prácticas humorísticas, como es el caso de las burlas y otras formas de agresión lúdica, tampoco encajan con los modelos de interacción «femeninos», que se basan en todo lo contrario, es decir, en la realización de actividades discursivas de carácter cooperativo que buscan la expresión de apoyo y la solidaridad de manera muy explícita, como contar problemas o experiencias personales negativas ${ }^{4}$.

1. LAKOFF, Robin. Language and woman's place. Text and commentaries. Editado por Mary Bucholtz. Oxford, Oxford University Press, 2004 (1975).

2. Ibíd., pp. 80-81.

3. LozANO DOMINGo, Irene. Lenguaje femenino, lenguaje masculino. ¿Condiciona nuestro sexo la forma de hablar? Madrid, Minerva, 1995.

4. ACUÑA FerReira, A. Virginia. «Complaint stories revisited. The 'masculine' performance of a 'feminine' genre in a conversation among Galician men». Sociolinguistic Studies 2.2 (2008), pp. 185-220. COATES, Jennifer. Women talk. Conversation between women friends. Oxford, Blackwell, 1996. COATES, Jennifer. Women, men and everyday talk. Londres, Palgrave, 2013. GüNTHNER, Susanne. «Complaint stories. Constructing emotional 
Sin embargo, desde la aparición de un interés específico por el humor desde una perspectiva de género, numerosos estudios han resaltado que este tipo de discurso también puede realizarse de forma cooperativa, de acuerdo con las características que se atribuyen a los modelos de interacción «femeninos». En un artículo pionero sobre este tema, Jenkins ${ }^{5}$ sostuvo que el humor de las mujeres no ha sido reconocido como tal porque presenta importantes diferencias con respecto al de los hombres: «up to now most things have been defined by men, including communication forms, so if women didn't do it like men, they didn't do it $»^{6}$. Basándose en su observación de las reuniones de un grupo de madres angloamericanas, esta autora destaca la narración frecuente de experiencias personales negativas en clave de humor, que servían para aliviar sentimientos de vergüenza y que buscaban la empatía de las interlocutoras $^{7}$. Desde su punto de vista, este tipo de historias constituyen una de las formas esenciales en que se manifiesta el humor de apoyo característico de las mujeres. Además, pone de relieve los comentarios que las participantes solían hacer para burlarse de los estereotipos sexistas que circulan en la sociedad, subrayando por tanto la función subversiva que cumplía el humor de las participantes en estas reuniones. Otros estudios señalan la importancia de estas dos formas de humor en el habla femenina; por una parte, se resalta que las mujeres cuentan historias humorísticas sobre experiencias personales negativas en mayor medida que los hombres ${ }^{8}$, en general, que el humor que expresa apoyo y que se realiza de forma colaborativa es más común en las

reciprocity among women», en Helga Kotthoff y Ruth Wodak (eds.): Communicating gender in context, Amsterdam, John Benjamins, 1997, pp. 179-218. Kendall, Shari y TANnEN, Deborah. «Discourse and gender», en Deborah Schiffrin, Deborah Tannen y Heidi E. Hamilton (eds.): The handbook of discourse analysis, Oxford, Blackwell, 2001, pp. 548-567. TANnEN, Deborah. You just don't understand. Women and men in conversation. Nueva York, William Morrow, 1990. TANnEN, Deborah. Género y discurso. Barcelona, Paidós, 1996.

5. JENKINS, Mercilee. «What's so funny? Joking among women», en Noelle Caskey, Sue Bremner, Birch Moonwomon y Noelle Caskey (eds.): Proceedings of the First Berkeley Women and Language Conference, University of California, Berkeley, Berkeley Women and Language Group, 185, pp. 131-151

6. Ibíd., p. 135.

7. Ibíd., p. 138-139.

8. ERVIN-TRIP, Susan y Martin D. Lampert. «Gender differences in the construction of humorous talk», en Kira Hall, Mary Bucholtz y Birch Moonwomon (eds.): Locating power. Proceedings of the Second Berkeley Women and Language Conference. Volume 1, Berkeley, CA, University of California, 1992, pp. 108-117. HAY, Jennifer. «Functions of humor in the conversations of men and women». Journal of Pragmatics 32 (2000), pp. 709-742. GEORGAKOPOUlOU, Alexandra. «Women, men and conversational narrative performances: Aspects of gender in Greek storytelling». Anthropological Linguistics 37 (1995), pp. 460-486. KotTHOFF, Helga. «Gender and joking: On the complexities of 
interacciones femeninas o mixtas, es decir, en acontecimientos comunicativos en los que participan mujeres ${ }^{9}$. Por otra parte, se hace hincapié en la utilización femenina del humor como una estrategia para desafiar y/o burlarse de las normas y/o estereotipos de género ${ }^{10}$, a veces incluso a través de prácticas estereotípicamente masculinas, como el humor sexual ${ }^{11}$. Acuña Ferreira ${ }^{12}$ aborda la realización de tipos de humor estereotípicamente masculino en conversaciones de jóvenes veinteañeras, como los chistes y las imitaciones, el empleo de lenguaje ordinario, o las bromas relacionadas con las borracheras, como formas de construcción de feminidades transgresoras.

Nuestro objetivo en este artículo es analizar la práctica de un tipo de humor que está asociado estereotípicamente con las mujeres y que sin embargo no parece haber sido abordado de forma empírica dentro de este campo: el humor que se produce en los cotilleos. En su artículo pionero sobre género y humor conversacional, Jenkins ${ }^{13}$ destacó que este es precisamente el único tipo de humor que se ha relacionado tradicionalmente con las mujeres, aunque las conversaciones femeninas que ella estudió no mostraban su práctica. Nuestros datos, sin embargo, incluyen conversaciones de jóvenes veinteañeras que contienen secuencias de cotilleo, en las que este tipo de habla se realiza desde una perspectiva humorística y/o burlona. Así pues, el objetivo más específico de este trabajo es analizar las características formales y funcio-

women's image politics in humourous narratives». Journal of Pragmatics 32 (2000), pp. 55-80.

9. HolmES, Janet. «Sharing a laugh: Pragmatic aspects of humor and gender in the workplace». Journal of Pragmatics 38 (2006), pp. 26-50.

10. KALČIK Susan. «... like Ann's gynecologist or the time I was almost raped: Personal narratives in women's rap groups». Journal of American Folklore 88 (1975), pp. 3-11. FARR, Marcia. «Echando relajo: Verbal art and gender among Mexicanas in Chicago», en Mary Bucholtz, A.C. Liang, Laurel A. Sutton y Caitling Hines (eds.): Cultural performances. Proceedings of the Third Berkeley Women and Language Conference, Berkeley, CA, University of California, 1994, pp. 168-186. FARR, Marcia. «Echando relajo: Verbal art and gender among Mexicanas in Chicago», en Mary Bucholtz, A.C. Liang, Laurel A. Sutton y Caitling Hines (eds.): Cultural performances. Proceedings of the Third Berkeley Women and Language Conference, Berkeley, CA, University of California, 1994, pp. 168186. HolmeS, Janet. «Sharing a laugh: Pragmatic aspects of humor and gender in the workplace». Op. cit. HOLMES, Janet. Gendered talk at work. Constructing gender identity through workplace discourse, Oxford, Blackwell, 2009.

11. ACUÑA FerReira, A. Virginia. Género y discurso. Las mujeres y los hombres en la interacción conversacional. Múnich, Lincom, 2009. GREENE, Rayna. «Magnolias grow in dirt: The bawdy lore of Southern women». Southern Exposure 4 (1977), pp. 29-33.

12. ACuña Ferreira, A. Virginia. Op. cit. ACuÑa FerReira, A. Virginia. «The humorous display of transgresor femininities: 'Sharing a laugh' in Spanish-Galician friendly talk among young women». Sociolinguistic Studies 6.1 (2012), 121-147.

13. JENKINS, Mercilee. Op. cit.

Feminismo/s 24, diciembre 2014, pp. 67-94 
nales de este tipo de humor, teniendo en cuenta las importantes funciones de competitividad que se han atribuido al cotilleo en general y al cotilleo de las mujeres en particular, desde diversas disciplinas. Antes de este análisis, por tanto, será importante revisar algunas investigaciones sobre el cotilleo que también tienen relevancia para el presente estudio.

\section{El cotilleo y las mujeres}

El cotilleo ha llamado la atención de los estudios de lengua y género, como forma de habla que ha estado estereotípicamente asociada con las mujeres en las sociedades occidentales ${ }^{14}$. Esta asociación se ha explicado principalmente como algo relacionado con la histórica división sexual de la esfera pública y privada ${ }^{15}$, aunque también se ha argumentado que los juicios peyorativos sobre el cotilleo de las mujeres responden a un intento de controlar la solidaridad femenina, considerada como una amenaza para la sociedad patriarcal ${ }^{16}$. Algunos estudios etnográficos han subrayado la importancia del cotilleo en la vida de las mujeres, como un canal ilegítimo de comunicación que posibilita el acceso femenino a la información relacionada con la vida pública ${ }^{17}$, y como un componente clave de la cultura oral femenina, esencial para la construcción de lazos de solidaridad entre las mujeres ${ }^{18}$.

En cambio, otros estudios descubren la utilización femenina del cotilleo como un arma para romper la armonía social y reestructurar las alianzas dentro del grupo. Las investigaciones de Goodwin ${ }^{19}$ analizan el cotilleo entre

14. Romaine, Suzanne. Communicating gender. Mahwah, NJ, Lawrence Erlbaum Associates, 1999.

15. SCOLlON, Ron y Suzanne SCOLlon. Intercultural communication. A discourse approach. Oxford, Blackwell, 1995.

16. RYSMAN, Alexander. «How the gossip became a woman». Journal of Communication 27 (1977), pp. 176-180. SPENDER, Dale. Man made language. Londres, Routledge y Kegan Paul, 1980.

17. HARDING, Susan. «Women and words in a Spanish village», en Rayna Reiter (ed.): Toward an anthropology of women, Nueva York, Monthly Review Press, 1975, pp. 283308. JONES, Deborah. "Gossip: Notes on women's oral culture». Women's Studies International Quarterly 3 (1980), pp. 193-198.

18. JONES, Deborah. «Gossip: Notes on Women's Oral Culture». Women's Studies International Quarterly 3 (1980), pp. 193-198.

19. Goodwin, Marjorie Harness. «He-said-she-said: Formal procedures for the construction of a gossip dispute activity». American Ethnologist 7 (1980), pp. 674-695. GOODWIN, Marjorie Harness. «Instigating: Storytelling as social process». American Ethnologist 9 (1982), pp. 799-819. Goodwin, Marjorie Harness. He-said-she-said: Talk as social organization among black children. Bloomington, Indiana University Press, 1990. GooDwin, Marjorie Harness. «Retellings, pretellings and hypothetical stories». Research on Language and Social Interaction 24 (1990/1991), pp. 263-276. 
niñas afroamericanas de un vecindario de Filadelfia como un proceso conflictivo, que se inicia con la producción de una historia instigadora, en la que una niña cuenta a otra lo que una tercera dijo de ella «a sus espaldas», propiciando el enfrentamiento entre ambas. Guendouzi ${ }^{20}$ resalta las funciones competitivas del bitching, un tipo de cotilleo fuertemente asociado con las mujeres que recibe esta etiqueta específica en inglés. Su análisis de esta práctica en conversaciones femeninas muestra que las participantes cooperan estrechamente entre sí para censurar moralmente a otras mujeres, en la medida en que su comportamiento supone una aberración desde el punto de vista de las normas de género. Por ello, esta autora pone de relieve la necesidad de prestar atención tanto al estilo como al contenido del discurso, y concluye que el bitching sirve para reivindicar capital social de forma competitiva mediante el refuerzo de normas sociales que establecen qué roles son aceptables para las mujeres; en sus propias palabras, el bitching puede definirse como «a form of gossip that involves comparative competition for social capital based on hegemonic images of femininity $»^{21}$.

\section{Tipos de cotilleo}

Desde la Psicología Social, Rosnow y Fine ${ }^{22}$ diferenciaron tres grandes funciones del cotilleo: la moralización, la información y el entretenimiento. En general, la bibliografía ha abordado ampliamente las dos primeras ${ }^{23}$, mientras que la función de entretenimiento ha recibido mucha menos atención. Como afirma Tholander ${ }^{24}$ «the idea that gossip is moralizing or norm enforcing is

20. GUENDOUZI, Jackie. «You'll think we're always bitching: the functions of cooperativity and competition in women's gossip». Discourse Studies 3.1 (2001), pp. 29-51.

21. Ibíd., p. 34.

22. ROSNOW, Ralph L. y FINE, Gary Alan. Rumor and gossip: The social psychology of hearsay. New York, Elsevier, 1976.

23. BERGMANN, Jörg R. Discreet indiscretions. The social organization of gossip. Nueva York: Aldine de Gruyter, 1993. Cox, Bruce A. «What is Hopi gossip about? Information management and Hopi factions». Man 5.1 (1970), pp. 88-98. EGGINS, Suzanne y SLADE, Diana. Analysing casual conversation. Londres, Continuum, 1997. GLUCKMAN, Max. «Gossip and scandal». Current Anthropology 4.3 (1963), pp. 307-316. GuENDOUZI, Jackie. «You'll think we're always bitching: the functions of cooperativity and competition in women's gossip». Discourse Studies 3.1 (2001), pp. 29-51. HALl, Joan Kelly. «Tengo una bomba»: The paralinguistic and linguistic conventions of the oral practice chismeando. Research on Language and Social Interaction 26.1 (1993), pp. 55-83. HAVILAND, John B. Gossip, reputation, and knowledge in Zinacantan. Chicago, University of Chicago Press, 1977.

24. THOLANDER, Michael. «Pupil's gossip as remedial action». Discourse Studies 5.1 (2003), pp. 101-129.

Feminismo/s 24, diciembre 2014, pp. 67-94 
perhaps the most accepted and widespread $\aleph^{25}$. Uno de los pocos trabajos que ha abordado el cotilleo orientado al entretenimiento es el capítulo de Morreall ${ }^{26}$ sobre la relación de este tipo de habla con el humor. Sin utilizar apoyo empírico, este autor sostiene que el cotilleo que se realiza desde un punto de vista humorístico es el «mejor» tipo de cotilleo, en la medida en que busca simplemente el entretenimiento, está libre de «mezquindad»y «malevolencia» y resulta incompatible con emociones negativas fuertes: «People cannot anger us or make us indignant, for instance, at the same time as they make us laugh $»^{27}$. En contraste, Tholander sugiere que incluso el cotilleo de entretenimiento tiende a ocultar intereses personales: «even if gossip might look light hearted and entertaining on the surface, it may be more serious at an underlying level» ${ }^{28}$.

Acuña Ferreira ${ }^{29}$ aborda el cotilleo en clave humorística de forma empírica, a través del análisis de extractos de conversaciones entre jóvenes veinteañeras gallegas. Desde el punto de vista funcional, los datos revelan que este tipo de cotilleo no solo sirve para entretenerse, sino que también conlleva otros beneficios, ya que puede actuar, por ejemplo, como una forma de obtener consuelo grupal ante un sentimiento de envidia, de incrementar la autoestima ante los problemas ajenos o de invalidar los éxitos de los demás. Estas funciones más implícitas encubren, a su vez, una competición comparativa entre las personas que participan en el cotilleo y aquella de la que se habla. Así, en este estudio se resalta que el cotilleo que se realiza de forma lúdica, humorística y burlona, como una forma de mofa indirecta, tampoco está libre de malicia y/o competitividad, de acuerdo con las argumentaciones en torno a las funciones de este tipo de habla que proceden de la Psicología Social ${ }^{30}$, y contrariamente, por tanto, a lo que sostenía Morrealli1 .

25. Ibíd., p. 104.

26. Morreall, John. «Gossip and humor», en Robert F. Goodman y Aaron Ben-Ze'ev A (eds.): Good gossip, Lawrence, University Press of Kansas, 1994, pp. 56-64.

27. Ibíd., p. 64.

28. THOLANDER, Michael. Op. cit., p. 105.

29. ACUÑA FERREIRA, A. Virginia. «Gossip as indirect mockery in friendly conversation: The social functions of 'sharing a laugh' at third parties». Discourse Studies 16.5 (2014), pp. 607-628.

30. Paine, Robert. 1967. What is gossip about? An alternate hypothesis. Man 2. 278-285. SulS, Jerry M. «Gossip as social comparison». Journal of Communication 27.1 (1977), pp. 164-168. WeRT, Sarah R. y SALOVEY, Peter. «A social comparison account of gossip». Review of General Psychology 8.2 (2004), pp. 122-137.

31. MORREALL, John. Op. cit. 


\section{Análisis de los datos}

En este apartado vamos a analizar cuatro fragmentos de dos conversaciones espontáneas procedentes del Corpus de Fala Bilingüe Galego/Castelán (Co. Fa.Bil) de la Universidad de Vigo $^{32}$, en las que participan estudiantes universitarias gallegas de poco más de veinte años que mantienen una relación de amistad. La actividad comunicativa se centra en cotillear de otras chicas y se desarrolla en la habitación de una residencia universitaria en un caso y en el servicio femenino de una cafetería en el otro; se trata de entornos discursivos «seguros», con un alto grado de intimidad, que permiten a las hablantes evitar o reducir las posibilidades de amenaza para su imagen positiva ${ }^{33}$. La grabación fue realizada en audio por uno de los miembros de cada grupo de amigas, con el propósito general de obtener datos conversacionales; ninguna de las participantes era consciente de que pudiese haber un interés más específico por la práctica de cotilleo como actividad comunicativa.

Las investigaciones sobre el cotilleo ofrecen distintas definiciones de este tipo de habla, como destaca Foster ${ }^{34}$, pero los casos que se van a analizar aquí se ajustan especialmente a la presentada por Taylor ${ }^{35}$, que define el «cotilleo prototípico» como habla entre dos o más personas acerca de la vida privada de una tercera "a sus espaldas», incluyendo sus amores, sus amistades, su matrimonio o su divorcio, «as well as her public activities insofar as these may throw light on her psychology or character ${ }^{36}$. Llamaremos Conversación A y Conversación B a cada una de las dos conversaciones de las que se han extraído los datos. El análisis se centrará en los rasgos formales y funcionales del discurso y se realizará turno por turno, haciendo hincapié en el carácter lúdico y humorístico de las contribuciones de las participantes.

32. El Corpus de Fala Bilingüe Galego/Castelán (Co.Fa.Bil) de la Universidad de Vigo está constituido por interacciones habladas que fueron grabadas en audio en contextos comunicativos «naturales» a través del método de la observación participante.

33. GUENDOUZI, Jackie. Op. Cit. El cotilleo se considera un acto comunicativo que amenaza la imagen positiva del hablante, en la medida en que se percibe socialmente de forma muy negativa.

34. FOSTER, Eric K. «Research on gossip: Taxonomy, methods, and future directions». Review of General Psychology 8.2 (2004), pp. 78-99.

35. TAYLOR, Gabriele. (1994). «Gossip as moral talk», en Robert F. Goodman y Aaron BenZe'ev (eds.): Good gossip. Lawrence, University Press of Kansas, 1994, pp. 34-46.

36. Ibíd, p. 34.

Feminismo/s 24, diciembre 2014, pp. 67-94 


\subsection{Conversación A: cotilleos en el servicio femenino}

En la Conversación A intervienen Nieves, Paula, María y Mónica ${ }^{37}$. El lugar de la interacción es el servicio femenino de una cafetería y la grabación tiene una duración total de casi treinta minutos. El Extracto 1 recoge la fase inicial de un cotilleo acerca de una chica que está saliendo con un chico al que todas conocen, según lo comentado previamente por una de las participantes. En esta fase inicial, que Eggins y Slade ${ }^{38}$ denominan third person focus, el discurso se centra en aportar información sobre la identidad de la chica:

\section{Extracto 1}

\begin{tabular}{|l|l|l|}
\hline 1274 & NIEVES & yo con- \\
\hline 1275 & & [yo conozco una Bea pero no sé si será la misma] \\
\hline 1276 & PAULA & [puta non petarda $(\mathrm{xx})$ ] \\
\hline 1277 & &.. \\
\hline 1278 & MARÍA & NO:: $\rightarrow$ \\
\hline 1279 & & ésta que andaba [mucho con::] \\
\hline 1280 & PAULA & $=\{[$ [p] a irmán de Pablo joder $\} \rightarrow$ \\
\hline 1281 & & $<1.5>$ \\
\hline 1282 & MARÍA & sabes? $=$ \\
\hline 1283 & NIEVES & $=$ que el padre está en \#localidad\#? \\
\hline 1284 & & $<$ asienten todas efusivamente $>$ \\
\hline 1285 & & el putón verbenero $\uparrow$ \\
\hline 1286 & & $<$ risas fuertes de todas $>$ \\
\hline 1287 & MÓNICA & es que es que sí $\uparrow=<$ riéndose $>$ \\
\hline 1288 & NIEVES & $=$ ya lo sé $\rightarrow=$ \\
\hline 1289 & MÓNICA & $=$ a ver si [lo sabe (mi madre)] \\
\hline 1290 & MARÍA & [jo:der $($ xx ] \\
\hline 1291 & NIEVES & [que está más sobada que un sobao Martínez] $=\rightarrow$ \\
\hline 1292 & LAURA & $=$ YO SIEMPRE DIGO $\rightarrow$ \\
\hline 1293 & & a ver cuánto [tiempo dura esto] \\
\hline 1294 & MARÍA & $\{[$ ac] [a] [QUE UN SOBAO QUÉ?] $\}=$ \\
\hline
\end{tabular}

37. Véase el Apéndice: Convenciones de Transcripción. Utilizamos seudónimos tanto en el caso de las participantes como en el de las terceras personas que son aludidas en la interacción.

38. EGGINS, Suzanne y Slade, Diana. Op. cit. 


\begin{tabular}{|l|l|l|}
\hline 1295 & NIEVES & $=\{[\mathrm{ac}]$ QUE ESTÁ MÁS SOBADA $\uparrow$ \\
\hline 1296 & & QUE UN SOBADO MARTÍNEZ $\} \rightarrow=$ \\
\hline 1297 & MARÍA & $=\mathrm{A}: \mathrm{H} \uparrow<$ risas de las demás> \\
\hline 1298 & & (te iba a decir yo) \\
\hline 1299 & & $<$ alguien tose $>$ \\
\hline 1300 & NIEVES & [yo lo siento mucho bueno] $\uparrow$ \\
\hline 1301 & MARÍA & {$[(\mathrm{xx})]=$} \\
\hline 1302 & NIEVES & $=$ si:: \\
\hline 1303 & & es muy amiga vuestra $\uparrow$ \\
\hline 1304 & & pero:: $[(\mathrm{xx})]$ \\
\hline 1305 & MÓNICA & $\{[$ ac] $[$ no no no $]\} \rightarrow=$ \\
\hline
\end{tabular}

Mientras Nieves expresa sus dudas acerca de si conoce a la chica aludida (líneas 1274-1275), Paula se refiere despectivamente a esta en gallego como unha petarda (línea 1276). En los turnos siguientes, María y Paula aportan información sobre quiénes son sus amistades y sus familiares (líneas 12781280), para ayudar a Nieves a darse cuenta de quién se trata. Tras una pausa (línea 1281), Nieves pregunta si su padre vive en determinada localidad (línea 1283), obtiene la confirmación de las demás (línea 1284), y entonces indica que ya sabe quién es la chica, identificándola como el putón verbenero (línea 1285), lo que provoca la risa de las demás (línea 1286). Mónica aprueba este comentario, entre risas (línea 1287), y Nieves se reafirma en que la conoce (línea 1288). En los siguientes turnos se produce un solapamiento de voces que impide comprender totalmente las intervenciones (líneas 1289-1291), pero sí se entiende una nueva aportación de Nieves, que utiliza un juego de palabras basado en el nombre y la marca de un producto de pastelería, que está más sobada que un sobao Martínez (línea 1291), para desplegar humor lingüístico y resaltar nuevamente la promiscuidad de la chica de manera burlona. Este chiste lingüístico pasa inicialmente desapercibido, porque está en superposición de voces, pero, en el siguiente turno, Laura expresa sus dudas acerca del futuro de la relación entre los dos jóvenes aludidos (líneas 1292-1293) y entonces María alza la voz para pedir a Nieves que repita su aportación previa (línea 1294). Esta repetición se produce en el turno inmediatamente posterior, con un volumen de voz también más elevado (líneas 1295-1296), y está seguida por una risa conjunta (línea 1297). Finalmente, Nieves pide disculpas por sus comentarios sobre la joven, preguntando indirectamente a sus compañeras si tienen algún tipo de amistad con ella (líneas 1300, 1303-1304), algo que Mónica niega inmediatamente (línea 1305). 
En resumen, este extracto recoge la fase inicial de un cotilleo en la que el habla se centra en despejar las dudas sobre la identidad de una de las terceras personas en las que se centra la actividad comunicativa. En este proceso, una de las participantes utiliza el descalificativo putón verbenero y realiza un chiste lingüístico, que está más sobada que un sobao Martínez, con varios fines: para indicar a sus compañeras que ya sabe quién es la tercera persona aludida, para mofarse de esta por su estilo de vida sexual y la reputación que tiene en este sentido, y para provocar la risa de las demás, contribuyendo al desarrollo del cotilleo en clave humorística. Las salidas graciosas de esta locutora reflejan la fuerza cultural del calificativo putón en la constitución y refuerzo del doble estándar sexual, que promueve la promiscuidad en los hombres, y en cambio la castiga en las mujeres ${ }^{39}$.

El humor desplegado se basa por tanto en un refuerzo de los discursos tradicionales sobre género y sexualidad y conlleva una agresión lúdica hacia la joven de la que se habla por la relación de pareja que ha iniciado con un chico. La realización de este tipo de ataque humorístico y la celebración del mismo por parte de todas las demás participantes a través de la risa sugieren un sentimiento común de envidia ante la noticia de esa relación y aportan un carácter competitivo y malicioso al discurso, ya que la descalificación peyorativa y burlona que se hace de la chica actúa como una forma de contrarrestar su «éxito» por esa relación. Desde el punto de vista del contenido, de la «sustancia» del discurso, en este extracto subyace una competitividad por las relaciones con los hombres; en los estudios específicos sobre género y sexualidad, este tipo de competitividad femenina es destacada como resultado de los discursos que establecen el matrimonio y la maternidad como máxima prioridad para las mujeres, y que de este modo fomentan la idea de «conseguir a un hombre» como algo primordial para ellas ${ }^{40}$.

En el Extracto 2, el cotilleo se encuentra en la fase que Eggins y Slade ${ }^{41}$ denominan substantiating behaviour, en la que «the speaker or speakers provide evidence or information which enables the participants to make a

39. CowIE, Celia y LeES, Sue. «Slags or drags». Feminist Review 9 (1981), pp. 17-31. LeES, Sue. Sugar and spice: Sexuality and adolescent girls. Londres, Penguin, 1986. WaLton, Marsha, WeATHERALl, Ann y JACKSON, Sue. «Romance and friendship in pre-teen stories about conflicts: "we decided that boys are not worth it». Discourse \& Society 13 (2002), pp. 673-689.

40. GRIFFIN, Christine. «Absences that matter: Constructions of sexuality in studies of young women's friendships». Feminism \& Psychology 10 (2000), pp. 227-245.

41. EgGins, Suzanne y SLADE, Diana. Op. cit. 
negative evaluation» ${ }^{42}$. Aquí el discurso se centra en el comportamiento de una chica que participó en un desfile de moda:

\section{Extracto 2}

\begin{tabular}{|c|c|c|}
\hline 1383 & LAURA & Y LO MEJOR NO FUE EN EL DESFILE $\rightarrow$ \\
\hline 1384 & & LO MEJOR FUE $\rightarrow$ \\
\hline 1385 & & QUE EN EL (probador) $\uparrow \uparrow$ \\
\hline 1386 & & $<1>$ \\
\hline 1387 & & la niña $\rightarrow$ \\
\hline 1388 & & todas dentro $\uparrow$ \\
\hline 1389 & & porque $\rightarrow$ \\
\hline 1390 & & $\{[\mathrm{p}][\mathrm{ac}]$ la mayoría no querían desfilar en bikini $\} \rightarrow$ \\
\hline 1391 & & ya fue ella allá $\uparrow$ \\
\hline 1392 & & $\mathrm{y}$ todas dentro $\uparrow$ \\
\hline 1393 & & así en plan $\rightarrow$ \\
\hline 1394 & & $\{[\mathrm{~b}]$ joder $\uparrow<$ voz falsa $>$ \\
\hline 1395 & & y esto no sé qué\} $\uparrow$ \\
\hline 1396 & & y en esto que ella se fue al primero $\rightarrow$ \\
\hline 1397 & & ya le quedaba como una patada en el culo $\uparrow$ \\
\hline 1398 & & y sale $\rightarrow$ \\
\hline 1399 & & (xx) así de los probadores $\uparrow$ \\
\hline 1400 & & <LAURA imita la salida de la chica de los probadores> \\
\hline 1401 & & $\{[a]$ QUÉ TAL?\} <burlonamente, voz cursi> \\
\hline 1402 & PAULA & he he he \\
\hline 1403 & NIEVES & y [y y::] \\
\hline 1404 & LAURA & {$[\mathrm{Y}$ TODAS $] \rightarrow=$} \\
\hline 1405 & NIEVES & $=\mathrm{y}$ todas $[(\mathrm{xx}) \mathrm{ya}] \rightarrow<$ entre risitas $>$ \\
\hline 1406 & LAURA & [he he he] \\
\hline 1407 & & sí:: <entre risas> \\
\hline 1408 & & $\mathrm{y}$ todas $\rightarrow$ \\
\hline 1409 & & [bue::no] $\rightarrow$ \\
\hline 1410 & PAULA & {$[\mathrm{NON}] \rightarrow$} \\
\hline
\end{tabular}

42. Ibíd., p. 285.

Feminismo/s 24, diciembre 2014, pp. 67-94 


\begin{tabular}{|l|l|l|}
\hline 1411 & & É [QUE EU NON CRITICO ISO] $\rightarrow$ \\
\hline 1412 & NIEVES & [porque claro NO:] \\
\hline 1413 & PAULA & EU [O QUE CRITICO FOI] $\uparrow$ \\
\hline 1414 & NIEVES & {$[$ NO: $(\mathrm{xx})$ no fijo] $\downarrow=$} \\
\hline 1415 & PAULA & $=\{[\mathrm{dc}]$ A FO:RMA DE DESFILAR $\} \rightarrow=$ \\
\hline 1416 & NIEVES & $=$ yo no es eso $\uparrow$ \\
\hline 1417 & & es que $=$ \\
\hline 1418 & PAULA & $=$ É QUE QUE IMPORTA [SE $(\mathrm{xx})]$ \\
\hline 1419 & NIEVES & {$[$ DIO EL CA::NTE] $\uparrow$} \\
\hline 1420 & & dio [el CA::NTE] $\rightarrow$ \\
\hline
\end{tabular}

En este extracto, Laura produce una anécdota sobre el comportamiento de la joven que es objeto del cotilleo durante un desfile de moda. Eggins y Slade ${ }^{43}$ explican que en las anécdotas se presenta una crisis o complicación que, a diferencia de lo que sucede en las narrativas ${ }^{44}$, no está seguida por una resolución, sino por una reacción: «rather than being explicitly resolved, the crisis is reacted to in some way: by an expression of amazement, frustration, emarrasement, humiliation, etc» ${ }^{45}$. El discurso de Laura en este extracto se ajusta a la estructura de este tipo de historias, según estas autoras. En primer lugar, la hablante desarrolla la fase de orientación, cuando señala explícitamente que la mayoría de las chicas que participaban en el desfile no querían desfilar en bikini y que estaban todas dentro (líneas 1388-1390), sugiriendo así que les avergonzaba hacerlo. Seguidamente, Laura repite todas dentro y recrea la voz de alguien que expresa una queja por la posibilidad de tener que desfilar en bikini, joder y esto no sé qué (líneas 1394-1395), utilizando el discurso referido directo como una demostración ${ }^{46}$ de esa actitud negativa o sentimiento de vergüenza aparentemente generalizado; en contraste, se indica que la chica que es objeto del cotilleo se dirigió a los probadores, ya fue ella allá (línea 1391), y en esto que ella se fue al primero (línea 1396), empleando el adverbio ya y el conector narrativo y en esto que para sugerir la rapidez o la naturalidad con la que lo hizo y al mismo tiempo para anunciar el inicio de la fase

43. EGGins, Suzanne y SLADE, Diana. Op. cit.

44. LABOV, William y WALETZKY, Joshua. «Narrative analysis», en June Helm (ed.): Essays on the verbal and visual arts. Seattle, University of Washington Press, 1967, pp. 12-44.

45. EgGins, Suzanne y SLAdE, Diana. Op. cit., p. 237.

46. Clark, Herbert y Gerrig, Richard. "Quotations as demonstrations». Language 66, (1990), pp. 764-805. 
de reconstrucción del acontecimiento destacable que constituye el corazón narrativo de las anécdotas ${ }^{47}$.

Finalmente, Laura se centra en este acontecimiento clave y describe el momento en el que la protagonista de la historia sale del probador con el bikini puesto, resaltando inicialmente lo mal que le quedaba, ya le quedaba como una patada en el culo (línea 1397), para después representar su actitud de orgullo y/o ilusión al exhibirse ante las demás, haciendo una imitación no verbal (líneas 1399-1400), y recreando su voz preguntando cómo le sentaba, qué tal (línea 1401), con rasgos prosódicos de estilización paródica ${ }^{48}$, que sirven para caracterizar burlonamente al personaje por su voz «cursi».

Paula se ríe (línea 1402) y a continuación se produce una lucha por el turno entre Nieves y Laura, que tratan de completar la historia de forma paralela, entre risas, representando la reacción de las demás chicas que participaban en el desfile ante la aparición de la protagonista en bikini (y demostrando por tanto que ambas conocían los hechos, líneas 1404-1409). Paula utiliza un volumen de voz más fuerte para llamar la atención de sus compañeras sobre su punto de vista ante la actuación de la «víctima» del cotilleo en ese desfile (líneas 1410-1411, 1413, 1415, 1418), pero su estrategia no tiene mucho éxito, ya que Nieves interviene paralelamente (líneas 1412, 1414, 14161417). Ambas participantes compiten por destacar su propia opinión sobre la persona que está en el foco de la actividad comunicativa, de manera que el discurso adquiere un carácter evaluativo. En las líneas 1419-1420, Nieves eleva también el volumen de voz para resaltar una evaluación explícita de los hechos basada en la expresión coloquial dar el cante (líneas 1419-1420), que utiliza para criticar el comportamiento que tuvo la chica en cuestión, en contraste con el de las demás participantes en el desfile. Este comentario evaluativo actúa como la coda de una anécdota divertida, en la que se presenta un acontecimiento destacable por lo ridículo que resulta, cuya reconstrucción está orientada a provocar la risa de la audiencia como reacción ${ }^{49}$, pero también a suscitar un discurso evaluativo crítico que es más específicamente característico del cotilleo.

47. EgGins, Suzanne y Slade, Diana. Op. cit.

48. BAKHTIN, Mikhail M. «Discourse in the novel», en Michael Holquist y Caryl Emerson (eds.): The dialogic imagination: Four essays. Austin: University of Texas Press, 1981, pp. 259-422. GÜNTHNER, Susanne. «Complaint stories. Constructing emotional reciprocity among women», en Helga Kotthoff y Ruth Wodak (eds.): Communicating gender in context, Amsterdam, John Benjamins, 1997, pp. 179-218.

49. EGgINS, Suzanne y SLADE, Diana. Op. cit., p. 237. 
Desde una perspectiva de género, el contenido de esta anécdota gira en torno a un mundo fuertemente feminizado como es el de la moda y los desfiles de modelos, que ejerce una poderosa influencia en la reafirmación de la belleza, de la apariencia física y del aspecto en general, como parte esencial del capital simbólico ${ }^{50}$ (Bourdieu, 1987) disponible para las mujeres en la sociedad contemporánea, en consonancia con los discursos de género de épocas pasadas. Como ha destacado Guendouzi: «the media still puts forward restricted images of femininity that require women to live up to social ideals such as the 'good mother', 'looking young', and having the 'ideal body shape' $»^{51}$. En este extracto, la joven de la que se habla participa como modelo en un desfile, y la realización de este rol la sitúa como una «mujer de éxito» o un «modelo de feminidad», desde el punto de vista de estos discursos que ensalzan la belleza y la apariencia física como valores femeninos. Sin embargo, las locutoras se centran en su actuación en ese desfile, a través de la producción de una anécdota humorística, para mofarse de ella y para criticarla por su falta de reparos ante la posibilidad de tener que desfilar en bikini, en contraste con la vergüenza que mostraban las demás chicas que participaban en el desfile, y por su actitud presuntuosa al exhibirse orgullosa con esta prenda. En esta crítica burlona subyace una reivindicación de otros símbolos de feminidad, como la modestia ${ }^{52}$ y el pudor o la vergüenza ${ }^{53}$, que sirve de contrapeso al «éxito» de la víctima de estas críticas por su participación como modelo en un desfile. Nótese que, además, se llegan a negar explícitamente sus cualidades físicas para realizar este rol (línea 1397). De forma similar a lo que veíamos en el Extracto 1, el discurso adquiere connotaciones de competitividad o rivalidad por un tipo de capital simbólico femenino que logra u ostenta la tercera persona que es objeto de la actividad comunicativa, pero que las participantes tratan de contrarrestar o de anular a través de la crítica y la mofa. En este sentido, la anécdota humorística que recoge este extracto cumple también, por tanto, una función competitiva.

\subsection{Conversación B: cotilleos en la habitación de la residencia}

En la conversación B intervienen Olga, Vanesa y Laura. La interacción se desarrolla en este caso en la habitación de una residencia universitaria y se basa casi exclusivamente en la práctica de cotilleo, durante los veinticinco minutos

50. BourdieU, Pierre. Language and symbolic power. Londres, Polity Press, 1987.

51. GuendouZI, Jackie. Op. Cit., p. 31.

52. MilLs, Sara. Gender and politeness. Cambridge, Cambridge University Press, 2003.

53. COATES, Jennifer. Women talk. Conversation between women friends. Op. Cit. COATES, Jennifer. Women, men and everyday talk. Op. Cit. 
que abarca aproximadamente la grabación. Además, este cotilleo se centra en una única persona, Cristina, otra estudiante universitaria que conocen bien las tres participantes, pero sobre todo Laura, ya que fue su compañera de piso en la misma residencia donde tiene lugar la conversación y además solía salir con ella. La actividad comunicativa se basa en las historias e informaciones que aporta Laura en relación con la forma de ser y de actuar de Cristina, sus costumbres y su vida personal, especialmente sus relaciones con los chicos, a veces por petición explícita de las demás y otras veces en respuesta a sus muestras de interés. El discurso se produce generalmente en clave humorística y/o burlona; por ejemplo, en el Extracto 3, Laura se queja por la frecuencia con la que Cristina solía hablar por teléfono con sus novios o amigos y representa paródicamente la forma en que lo hacía:

\section{Extracto 3}

\begin{tabular}{|l|l|l|}
\hline 518 & LAURA & $=$ andaba todo el día $\uparrow$ \\
\hline 519 & & $<1.5>$ \\
\hline 520 & & es que $\rightarrow$ \\
\hline 521 & & era una cosa $\uparrow$ \\
\hline 522 & &.. \\
\hline 523 & & llamaba uno $\uparrow$ \\
\hline 524 & & colgaba $\rightarrow$ \\
\hline 525 & &.. \\
\hline 526 & & llamaba otro $\uparrow$ \\
\hline 527 & & a lo mejor se pasaba $\rightarrow$ \\
\hline 528 & & toda la noche $\uparrow$ \\
\hline 529 & & hablando por teléfono $\rightarrow=$ \\
\hline 530 & VANESA & $=\{[$ ac] ay eso sí:: \\
\hline 531 & & es verdá $\rightarrow$ \\
\hline 532 & & [eso sí porque él estuvo aquí]\} $\uparrow$ \\
\hline 533 & LAURA & [y cada mariconada::] $\uparrow=$ \\
\hline 534 & VANESA & $=$ = y la llamaron cada [cinco minutos] $\rightarrow$ \\
\hline 535 & LAURA & $\{[$ [p] [y cada mari-]\} $\uparrow$ \\
\hline 536 & & es que soltaba cada maricona:da $\uparrow$ \\
\hline 537 & & .. \\
\hline 538 & & $\{[$ a] mi amo:::r $\uparrow<$ burlonamente, voz cursi $>$ \\
\hline
\end{tabular}




\begin{tabular}{|l|l|l|}
\hline 539 & & mi peluSI::to $\uparrow$ \\
\hline 540 & & mi no sé qué $\} \rightarrow=$ \\
\hline 541 & VANESA & $=$ he he he $=$ \\
\hline 542 & LAURA & $=\{[$ ac $]$ AL PORTUGUÉS LO LLAMABA MIMOSÍN $\} \rightarrow=$ \\
\hline 543 & VANESA & $=$ he he he $=$ \\
\hline 544 & OLGA & $=$ he he he $=$ \\
\hline 545 & LAURA & $=$ mira $\rightarrow=<$ entre risas $>$ \\
\hline 546 & VANESA & $=$ he he he $=$ \\
\hline 547 & LAURA & $=$ yo de verdá $\uparrow$ \\
\hline 548 & & que $\rightarrow$ \\
\hline
\end{tabular}

En este extracto, Laura describe la costumbre de Cristina de hablar por teléfono con chicos de manera crítica (líneas 518-529), como algo desmesurado y molesto, usando formulaciones de casos extremos ${ }^{54}$ como todo el día (línea 518) y toda la noche (línea 528), y especificando que estas charlas telefónicas se mantenían una detrás de otra (líneas 523-526). Vanesa confirma la veracidad de esta información, señalando que ella misma observó también un comportamiento similar por parte de Cristina (líneas 530-532, 534), y Laura prosigue para criticar la forma en que hablaba con esos chicos, evaluando despectivamente las cosas que les decía como una mariconada (líneas 533, 535536). En las líneas 538-540, Laura introduce discurso referido dramatizado ${ }^{55}$, en el que se incluyen recursos de estilización paródica ${ }^{56}$, para mofarse del estilo de habla cursi que Mónica empleaba en esas conversaciones; utiliza un tono más agudo para crear una voz en falsetto y un tono burlón al reconstruir directamente sus palabras o expresiones para dirigirse a ellos, mi amor (línea 538) y mi pelusito (línea 539), con alargamiento vocálico en ambos casos. Esta representación paródica provoca la risa de Vanesa (línea 541). Seguidamente, Laura eleva el volumen de voz para llamar la atención de sus compañeras sobre el apelativo que Cristina empleaba para dirigirse cariñosamente a un hombre portugués con el que tuvo una relación, al portugués lo llamaba Mimosín (línea 542); el énfasis en el uso de este apelativo actúa como un recurso de evaluación implícita que refuerza la representación previa de Cristina como una chica cursi. Vanesa y Olga despliegan afiliación con esta mofa a través de la risa (líneas 543-544).

54. POMERANTZ, Anita. «Extreme case formulations: A way of legitimizing claims». Human Studies 9 (1986), pp. 219-229.

55. BERGMANN, Jörg R. Op. cit.

56. BAKHTIN, Mikhail M. Op. cit. GÜNTHNER, Susanne. Op. cit. 
Como en la anécdota humorística que recogía el Extracto 2, aquí se observa cómo se utiliza la reconstrucción paródica del discurso de la «víctima» del cotilleo, como estrategia para mofarse indirectamente de ella (véase también Acuña Ferreira) ${ }^{57}$; en este caso, la mofa se basa únicamente en esta estrategia, es decir, el discurso se centra en ridiculizar a Cristina por su forma de hablar por teléfono con los chicos con los que se relacionaba y por la frecuencia con la que lo hacía. El foco de la crítica y de la mofa hacia la persona que es objeto de la actividad comunicativa está de nuevo, como ocurría en el Extracto 1, en las relaciones sexuales/amorosas con los hombres, lo que saca a relucir nuevamente una competitividad subyacente por esta cuestión como fruto de los discursos de género predominantes, tal y como destacábamos previamente. En el presente extracto, Cristina aparece posicionada como una mujer exitosa en el terreno amoroso, en la medida en que recibe numerosas llamadas de hombres y aparentemente tiene o ha tenido varios novios, pero también recibe de manera indirecta, a través del formato del cotilleo, un ataque humorístico por la forma en que se comunicaba con ellos. La representación jocosa de su estilo de habla y de sus expresiones sirve para trivializar o menospreciar ese aparente éxito con los hombres y en este sentido el discurso adquiere un carácter competitivo.

Lo cierto es que gran parte del cotilleo sobre Cristina en esta conversación tiene que ver con sus asuntos amorosos: la apariencia física de sus novios, quiénes son o con quiénes ha tenido una relación, su forma de comportarse con ellos, etc. En el Extracto 4 se recoge una anécdota humorística contada por Laura, en la que se pone de relieve la actitud de Cristina en los bares nocturnos a los que ambas, junto con otras chicas, solían acudir para divertirse:

\section{Extracto 4}

\begin{tabular}{|l|l|l|}
\hline 846 & LAURA & una vez estábamos con unos viejos que se nos pegaran $\uparrow$ \\
\hline 847 & & y la tía $\rightarrow$ \\
\hline 848 & & he he he \\
\hline 849 & & y yo $\uparrow$ \\
\hline 850 & & estaba toda puesta $\uparrow$ \\
\hline 851 & & $\{[$ a] hey $\uparrow<$ <entre risas> \\
\hline 852 & & queréis conocer a Cristi::na?\} \\
\hline
\end{tabular}

57. ACuña Ferreira, A. Virginia. «Gossip as indirect mockery: The social functions of 'sharing a laugh' at third parties». Op. cit. 


\begin{tabular}{|l|l|l|}
\hline 853 & & he he he \\
\hline 854 & & y Cristina $\rightarrow$ \\
\hline 855 & & $<$ LAURA hace una imitación $>$ \\
\hline 856 & VANESA & [he he he] \\
\hline 857 & LAURA & [he he he] \\
\hline 858 & & y el tío $\rightarrow$ \\
\hline 859 & & y el viejo $\rightarrow$ \\
\hline 860 & & hoy no pescas nada mujer $\uparrow$ \\
\hline 861 & & no sé qué $=<$ entre risas $>$ \\
\hline 862 & VANESA & $=\{[$ ac] $[$ CÓ::MO?] $\}$ \\
\hline 863 & LAURA & [y decía] $<$ entre risas $>$ \\
\hline 864 & & hoy no pescas nada $\rightarrow$ \\
\hline 865 & & $($ xx $)$ \\
\hline 866 & & y la tía $\rightarrow$ \\
\hline 867 & & $\{[$ a] no $\rightarrow$ \\
\hline 868 & & no hay suerte $\rightarrow$ \\
\hline 869 & & $($ xx $\}$ \\
\hline 870 & & y yo $\rightarrow$ \\
\hline 871 & & va::le $\rightarrow$ \\
\hline 872 & VANESA & he he he \\
\hline 873 & LAURA & he he he \\
\hline 874 & & mira $\rightarrow$ \\
\hline 875 & & yo de verdad que aún lo pasé acojonante $\rightarrow<$ entre risas $>$ \\
\hline 876 & & también es cierto $\downarrow=$ \\
\hline 877 & VANESA & $=[$ sí] $<$ entre risas $>$ \\
\hline 878 & LAURA & {$[$ [xx)] todo lo que quieras $\uparrow$} \\
\hline 879 & & pero me lo pasaba acojoNAN:te $\rightarrow=$ \\
\hline 880 & VANESA & $=$ he he he $=$ \\
\hline
\end{tabular}

Como orientación de la anécdota ${ }^{58}$, Laura señala que ella y su grupo de amigas se encontraban acompañadas por unos hombres, a los que se refiere despectivamente como viejos (línea 846). Tras un falso comienzo, seguido por una risa (líneas 847-848), la hablante especifica que ella misma estaba toda puesta (línea 850), para sugerir que tenía ganas de gastar bromas, que es justamente

58. EgGins, Suzanne y SLADE, Diana. Op. cit.

Feminismo/s 24, diciembre 2014, pp. 67-94 
lo que se reconstruye a continuación. Como acontecimiento destacable de esta anécdota, Laura reconstruye en estilo directo cómo ella misma propone a esos hombres que conozcan a Cristina, recreando una voz en falsetto a través del uso de un tono más agudo, hey queréis conocer a Cristina (líneas 851-852); de nuevo, se ríe (línea 853), para resaltar así, implícitamente, lo divertida que resultaba (o iba a resultar) la situación, y seguidamente hace una imitación no verbal de Cristina en respuesta a esa propuesta de presentación, que provoca la risa de Vanesa, además de la suya propia (líneas 854-857). A juzgar por cómo se sigue desarrollando la anécdota, cabe suponer que esta imitación representa a Cristina mostrando una rápida disposición por conocer a esos hombres; así, en las siguientes líneas (858-861), Laura reconstruye en estilo directo cómo uno de estos personajes masculinos se dirige a Cristina para decirle hoy no pescas nada mujer (línea 860); de este modo, el hombre trata de ponerla en ridículo ante todos, insinuando con sorna que su búsqueda de hombres era evidente y que no iba a lograr su objetivo.

Vanesa pide una aclaración o repetición del comentario realizado por este personaje a través de una pregunta cuyos rasgos prosódicos, aceleración del tempo y aumento del volumen, expresan asombro o incredulidad (línea 862), de manera que Laura lo repite (líneas 863-865), y a continuación reproduce directamente, utilizando un tono de voz más agudo, la respuesta de Cristina a ese comentario, expresando resignación, no no hay suerte (líneas 866869). El discurso referido directo que recogen las líneas 870-871, y yo vale, puede interpretarse como inner speech ${ }^{59}$, es decir, como una recreación del pensamiento que la propia Laura tuvo en ese momento, y que parece expresar satisfacción ante la burla hacia Cristina, que en parte fue propiciada por ella misma, al presentarle a esos hombres que estaban en el local. Esta expresión funciona como la reacción de la anécdota, junto con la risa de Vanesa que se produce inmediatamente después (línea 872). Finalmente, la anécdota incluye una coda, en la que Laura se ríe y resalta lo bien que se lo pasaba al salir con Cristina, mira yo de verdad que aún lo pasé acojonante (líneas 874876, 878-879), mientras Vanesa sigue riéndose y expresa acuerdo con esta valoración final de la broma (líneas 877, 880).

Una vez más, en esta anécdota se pone así de relieve la idea de «conseguir a un hombre» como meta de las mujeres y como origen de competición o rivalidad entre ellas. En este caso, además, se hace referencia concreta y explícita a esta idea con el uso coloquial del verbo pescar por parte del personaje

59. TANNEN, Deborah. Talking voices. Repetition, dialogue and imagery in conversational discourse. Cambridge, Cambridge University Press, 1989.

Feminismo/s 24, diciembre 2014, pp. 67-94 
masculino que se burla de Cristina, la «víctima» de los cotilleos en esta Conversación $B$, precisamente por perseguir este objetivo, aparentemente sin éxito. La anécdota se basa en la reconstrucción de la burla que se hizo directamente de ella en aquel momento por ese motivo, incluyendo señales de que fue instigada, propiciada o buscada por la participante que cuenta lo ocurrido como otro personaje dentro del mundo de la historia. Al contar la anécdota, Laura se burla nuevamente de Cristina, pero ahora de forma indirecta, «saboreando» su triunfo al lograr que fuese ridiculizada y obteniendo, además, la complicidad de su interlocutora, Vanesa, a través de la risa. En los extractos anteriores, las mofas y las agresiones lúdicas realizadas indirectamente a través del cotilleo trivializaban o menospreciaban un éxito de la joven aludida desde el punto de vista de los ideales de feminidad; en este caso, sin embargo, el discurso humorístico desplegado supone la celebración de un fracaso, que acentúa de forma similar una rivalidad o competitividad subyacente.

\section{Conclusiones}

En este artículo se han analizado las características formales y funcionales del humor en la práctica de cotilleo entre jóvenes veinteañeras. Frente a la caracterización general del humor de las mujeres en términos de cooperación, solidaridad y subversión del género en estudios previos, en nuestro análisis se han puesto de relieve las funciones competitivas que realiza este tipo de discurso de manera encubierta o subyacente y su papel en el mantenimiento y refuerzo de valores dominantes de feminidad o formas de capital simbólico femenino como el aspecto y las relaciones sexuales/amorosas con los hombres. Las «víctimas» de los cotilleos analizados aparecen establecidas como tales en la medida en que lograron o tratan de lograr una de estas formas de capital simbólico femenino, pero el discurso sobre ellas se centra en criticarlas desde una perspectiva burlona por este motivo, a través de salidas graciosas que las etiquetan de forma despectiva, de anécdotas humorísticas y/o de representaciones paródicas de su estilo de habla. Este proceso incluye a veces una reivindicación implícita de otros valores tradicionalmente asociados con la feminidad, como el rechazo a la promiscuidad, la modestia o el pudor.

Una de las funciones más obvias del discurso en los extractos analizados es sin duda el entretenimiento y la idea de «compartir unas risas» a través de la interacción comunicativa; sin embargo, en nuestro análisis se ha demostrado que este divertimiento se basa en la ridiculización de la tercera persona que es objeto del habla y que esta mofa o burla indirecta que se hace de ella sirve además para menospreciar sus éxitos o celebrar sus fracasos en relación con esos dos valores de feminidad antes señalados: el aspecto y las relaciones 
con los hombres. Esta función más implícita de las formas de discurso humorístico que se han abordado refuerza las tesis defendidas por estudios previos sobre la competitividad subyacente en la práctica de cotilleo, especialmente de aquellos que proceden de la Psicología Social ${ }^{60}$; más concretamente, nuestro análisis apoya las conclusiones de Guendouzi sobre el bitching «as a means of competing for 'peer validated' versions of femininity in the social marketplace ${ }^{61}$ y sobre la importancia de que los estudios sobre género y discurso tengan no solamente en cuenta el estilo de organización del discurso, sino también la sustancia. A diferencia de los extractos conversacionales analizados por esta autora, en los que el cotilleo se realiza con «seriedad», y desde una perspectiva abiertamente moralizante, nuestros datos pueden interpretarse como ejemplos de una forma de bitching humorístico, en el que esa competición es expresada a través de la mofa o de la burla ${ }^{62}$.

Las funciones competitivas que encubre tanto el bitching moralizante como la variedad lúdica/humorística que aquí se ha resaltado tienen importantes implicaciones para los estudios sobre género y discurso, que han enfatizado el estilo cooperativo y la búsqueda de apoyo y solidaridad como rasgos característicos del habla de las mujeres, incluyendo sus prácticas humorísticas, mientras que los estilos de interacción competitivos han sido relacionados casi exclusivamente con el habla de los hombres y con la masculinidad. La práctica del bitching y del cotilleo humorístico como mofa indirecta pone de relieve los componentes competitivos que también puede incluir el habla entre mujeres, aunque en un nivel más profundo, así como el papel que ejercen a este respecto los valores de feminidad que han establecido y continúan fomentando las ideologías de género predominantes. En el fondo, el modo encubierto o indirecto en que se manifiestan estas formas de competición en la conversación entre mujeres puede interpretarse como un refuerzo de la relación más directa que se establece entre competición y masculinidad, es decir, como un factor que reafirma la idea de que competir abiertamente es un comportamiento que se considera aceptable (o incluso deseable) en los hombres, pero no en las mujeres. En este sentido, este tipo de prácticas discursivas pueden verse entonces como un recurso o estrategia especialmente útil para las mujeres, en la medida en que les permite competir entre sí de manera más aceptable. Aunque los discursos de género no incluyen o no suelen incluir la competición como ideal de feminidad, puede concluirse que al mismo tiempo la fomentan, de forma un tanto paradójica, en torno a valores como el aspecto

60. Paine, Robert. Op. cit. SULS, Jerry M. Op. cit. Wert, Sarah R. y SAlovey, Peter. Op. cit. 61. GuENDOUZI, Jackie. Op. cit., p. 17.

62. Véase también ACUÑa FerREIRA, A. Virginia. Op. cit.

Feminismo/s 24, diciembre 2014, pp. 67-94 
y las relaciones con los hombres, tal y como se ha puesto de manifiesto en nuestro análisis.

\section{Referencias bibliográficas}

ACUÑA FerreirA, A. Virginia. «Complaint stories revisited. The 'masculine' performance of a 'feminine' genre in a conversation among Galician men». Sociolinguistic Studies 2.2 (2008), pp. 185-220.

ACUÑa FerReira, A. Virginia. Género y discurso. Las mujeres y los hombres en la interacción conversacional. Múnich, Lincom, 2009.

ACUÑA FERREIRA, A. Virginia. «The humorous display of transgresor femininities: 'Sharing a laugh' in Spanish-Galician friendly talk among young women». Sociolinguistic Studies 6.1 (2012), 121-147.

ACUÑa FERREIRA, A. Virginia. «Gossip as indirect mockery in friendly conversation: The social functions of 'sharing a laugh' at third parties». Discourse Studies 16.5 (2014), pp. 607-628.

BAKHTIN, Mikhail M. «Discourse in the novel», en Michael Holquist y Caryl Emerson (eds.): The dialogic imagination: Four essays. Austin: University of Texas Press, 1981, pp. 259-422.

Bergmann, Jörg R. Discreet indiscretions. The social organization of gossip. Nueva York: Aldine de Gruyter, 1993.

BOXER, D. y CORTÉS-CONDE, Florencia. «From bonding to biting: Conversational joking and identity display». Journal of Pragmatics 27 (1997), pp. 275-294.

Bourdieu, Pierre. Language and symbolic power. Londres, Polity Press, 1987.

CLARK, Herbert y GERRIG, Richard. «Quotations as demonstrations». Language 66, (1990), pp. 764-805.

COATES, Jennifer. Women talk. Conversation between women friends. Oxford, Blackwell, 1996.

COATES, Jennifer. Women, men and everyday talk. Londres, Palgrave, 2013.

Cox, Bruce A. «What is Hopi gossip about? Information management and Hopi factions». Man 5.1 (1970), pp. 88-98.

COWIE, Celia y LEES, Sue. «Slags or drags». Feminist Review 9 (1981), pp. 17-31.

EDER, Donna. «Go get ya a French! Romantic and sexual teasing among adolescent girls», en Deborah Tannen (ed.): Gender and conversational interaction. Nueva York, Oxford University Press, 1993, pp. 17-31.

EGGINS, Suzanne y SLADE, Diana. Analysing casual conversation. Londres, Continuum, 1997.

ERVIN-TRIP, Susan y Martin D. Lampert. «Gender differences in the construction of humorous talk», en Kira Hall, Mary Bucholtz y Birch Moonwomon (eds.): Locating power. Proceedings of the Second Berkeley Women and Language Conference. Volume 1, Berkeley, CA, University of California, 1992, pp. 108-117. 
FARR, Marcia. «Echando relajo: Verbal art and gender among Mexicanas in Chicago», en Mary Bucholtz, A.C. Liang, Laurel A. Sutton y Caitling Hines (eds.): Cultural performances. Proceedings of the Third Berkeley Women and Language Conference, Berkeley, CA, University of California, 1994, pp. 168-186.

FOSTER, Eric K. «Research on gossip: Taxonomy, methods, and future directions». Review of General Psychology 8.2 (2004), pp. 78-99.

GEORGAKOPOUlOU, Alexandra. «Women, men and conversational narrative performances: Aspects of gender in Greek storytelling». Anthropological Linguistics 37 (1995), pp. 460-486.

GluCKMAn, Max. «Gossip and scandal». Current Anthropology 4.3 (1963), pp. 307-316.

GoodwIN, Marjorie Harness. «He-said-she-said: Formal procedures for the construction of a gossip dispute activity». American Ethnologist 7 (1980), pp. 674-695.

GoodwIn, Marjorie Harness. «Instigating: Storytelling as social process». American Ethnologist 9 (1982), pp. 799-819.

Goodwin, Marjorie Harness. He-said-she-said: Talk as social organization among black children. Bloomington, Indiana University Press, 1990.

GoodwIN, Marjorie Harness. «Retellings, pretellings and hypothetical stories». Research on Language and Social Interaction 24 (1990/1991), pp. 263-276.

GREENE, Rayna. «Magnolias grow in dirt: The bawdy lore of Southern women». Southern Exposure 4 (1977), pp. 29-33.

GRIFFIN, Christine. «Absences that matter: Constructions of sexuality in studies of young women's friendships». Feminism \& Psychology 10 (2000), pp. 227-245.

GUENDOUZI, Jackie. «You'll think we're always bitching: the functions of cooperativity and competition in women's gossip». Discourse Studies 3.1 (2001), pp. 29-51.

GÜNTHNER, Susanne. «Complaint stories. Constructing emotional reciprocity among women», en Helga Kotthoff y Ruth Wodak (eds.): Communicating gender in context, Amsterdam, John Benjamins, 1997, pp. 179-218.

HAY, Jennifer. «Functions of humor in the conversations of men and women». Journal of Pragmatics 32 (2000), pp. 709-742.

HALL, Joan Kelly. «Tengo una bomba»: The paralinguistic and linguistic conventions of the oral practice chismeando. Research on Language and Social Interaction 26.1 (1993), pp. 55-83.

HARDING, Susan. «Women and words in a Spanish village», en Rayna Reiter (ed.): Toward an anthropology of women, Nueva York, Monthly Review Press, 1975, pp. 283-308.

HaVILAND, John B. Gossip, reputation, and knowledge in Zinacantan. Chicago, University of Chicago Press, 1977. 
HolmeS, Janet. «Sharing a laugh: Pragmatic aspects of humor and gender in the workplace». Journal of Pragmatics 38 (2006), pp. 26-50.

Holmes, Janet. Gendered talk at work. Constructing gender identity through workplace discourse, Oxford, Blackwell, 2009.

JENKInS, Mercilee. "What's so funny? Joking among women», en Noelle Caskey, Sue Bremner, Birch Moonwomon y Noelle Caskey (eds.): Proceedings of the First Berkeley Women and Language Conference, University of California, Berkeley, Berkeley Women and Language Group, 185, pp. 131-151

JONES, Deborah. «Gossip: Notes on women's oral culture». Women's Studies International Quarterly 3 (1980), pp. 193-198.

KALČIK Susan.. «... like Ann's gynecologist or the time I was almost raped: Personal narratives in women's rap groups». Journal of American Folklore 88 (1975), pp. 3-11.

Kendall, Shari y TANNEN, Deborah. «Discourse and gender», en Deborah Schiffrin, Deborah Tannen y Heidi E. Hamilton (eds.): The handbook of discourse analysis, Oxford, Blackwell, 2001, pp. 548-567.

KotтhofF, Helga. «Gender and joking: On the complexities of women's image politics in humourous narratives». Journal of Pragmatics 32 (2000), pp. 55-80.

LAKOFF, Robin. Language and woman's place. Text and commentaries. Editado por Mary Bucholtz. Oxford, Oxford University Press, 2004 (1975).

LEES, Sue. Sugar and spice: Sexuality and adolescent girls. Londres, Penguin, 1986. LABOV, William y WALETZKY, Joshua. «Narrative analysis», en June Helm (ed.): Essays on the verbal and visual arts. Seattle, University of Washington Press, 1967, pp. 12-44.

LozAno Domingo, Irene. Lenguaje femenino, lenguaje masculino. ¿Condiciona nuestro sexo la forma de hablar? Madrid, Minerva, 1995.

Mills, Sara. Gender and politeness. Cambridge, Cambridge University Press, 2003.

MorreALl, John. «Gossip and humor», en Robert F. Goodman y Aaron Ben-Ze'ev A (eds.): Good gossip, Lawrence, University Press of Kansas, 1994, pp. 56-64. PAINE, Robert. «What is gossip about? An alternative hypothesis». Man 2 (1967), pp. 278-285.

POMERANTZ, Anita. «Extreme case formulations: A way of legitimizing claims». Human Studies 9 (1986), pp. 219-229.

RodríGUEZ-YÁÑEZ, Xoán P. y CASARES, Håkan. «The Corpus of Galician/Spanish Bilingual Speech of the University of Vigo: Codes tagging and automatic annotation». Estudios de Sociolingüística 3.2 y 4.1 (2002/2003), pp. 359-382.

Romaine, Suzanne. Communicating gender. Mahwah, NJ, Lawrence Erlbaum Associates, 1999.

RosnOw, Ralph L. y FINE, Gary Alan. Rumor and gossip: The social psychology of hearsay. New York, Elsevier, 1976. 
RYSMAN, Alexander. «How the gossip became a woman». Journal of Communication 27 (1977), pp. 176-180.

SPENDER, Dale. Man made language. Londres, Routledge y Kegan Paul, 1980.

SCOllon, Ron y Suzanne SCOllon. Intercultural communication. A discourse approach. Oxford, Blackwell, 1995.

Suls, Jerry M. «Gossip as social comparison». Journal of Communication 27.1 (1977), pp. 164-168.

TANnEN, Deborah. Talking voices. Repetition, dialogue and imagery in conversational discourse. Cambridge, Cambridge University Press, 1989.

TANEN, Deborah. You just don't understand. Women and men in conversation. Nueva York, William Morrow, 1990.

TANnen, Deborah. Género y discurso. Barcelona, Paidós, 1996.

TAYLOR, Gabriele. (1994). «Gossip as moral talk», en Robert F. Goodman y Aaron Ben-Ze'ev (eds.): Good gossip. Lawrence, University Press of Kansas, 1994, pp. 34-46.

THOLANDER, Michael. «Pupil's gossip as remedial action». Discourse Studies. 5.1 (2003), pp. 101-129.

Walton, Marsha, Weatherall, Ann y Jackson, Sue. «Romance and friendship in pre-teen stories about conflicts: «we decided that boys are not worth it». Discourse E Society 13 (2002), pp. 673-689.

WerT, Sarah R. y SAlOvey, Peter. «A social comparison account of gossip». Review of General Psychology 8.2 (2004), pp. 122-137.

\section{Apéndice: convenciones de transcripción}

\begin{tabular}{|l|l|}
\hline Letra redonda & Español \\
\hline Letra negrita & Gallego \\
\hline Línea de transcripción & Unidad de entonación \\
\hline$\uparrow$ & Entonación final ascendente \\
\hline$\downarrow$ & Entonación final descendente \\
\hline$\rightarrow$ & Entonación final más o menos sostenida \\
\hline$?$ & Entonación típicamente interrogativa \\
\hline.. & Micropausa (menos de un segundo) \\
\hline$<3>$ & Pausa medida en número de segundos \\
\hline o sea:: & Sonido alargado \\
\hline la lo- & Sonido truncado \\
\hline $\begin{array}{l}\text { allí es mucho eh? }= \\
=\text { ya ves }\end{array}$ & Encadenamiento de voces \\
\hline & \\
\hline
\end{tabular}

Feminismo/s 24, diciembre 2014, pp. 67-94 


\begin{tabular}{|l|l|}
\hline $\begin{array}{l}\text { y tú te estabas [meando] } \\
\text { [es que está] tres horas }\end{array}$ & Superposición de voces \\
\hline (parece $)$ & Transcripción dudosa \\
\hline$(\mathrm{xx})$ & Segmento ininteligible \\
\hline he he he & Risa \\
\hline LETRAS MAYÚSCULAS & Volumen relativamente fortis \\
\hline\{\} & Segmento al que afecta el fenómeno \\
\hline$\{[p]\}$ & Volumen relativamente piano \\
\hline$\{[a c]\}$ & Tempo relativamente acelerado \\
\hline$\{[a]\}$ & Tono más agudo \\
\hline$<$ riéndose $>$ & Comentarios adicionales \\
\hline
\end{tabular}

Feminismo/s 24, diciembre 2014, pp. 67-94 